\title{
Comparative Metabolic Modeling of Multiple Sulfate-reducing Prokaryotes Reveals Versatile Energy Conservation Mechanisms
}

\author{
Wentao TANG ${ }^{1}$, Tian-Wei Hao ${ }^{2}$, and Guang-Hao Chen ${ }^{1}$ \\ ${ }^{1}$ Hong Kong University of Science and Technology \\ ${ }^{2}$ University of Macau Faculty of Science and Technology
}

August 28, 2020

\begin{abstract}
Sulfate-reducing prokaryotes (SRPs) are crucial participants in the cycling of sulfur, carbon, and various metals in the natural environment and in engineered systems. Despite recent advances in genetics and molecular biology bringing a huge amount of information about the energy metabolism of SRPs, little effort has been made to link this important information with their biotechnological studies. This study aims to construct multiple metabolic models of SRPs that systematically compile genomic, genetic, biochemical, and molecular information about SRPs to study their energy metabolism. Pan-genome analysis is conducted to compare the genomes of SRPs, from which a list of orthologous genes related to central and energy metabolism is obtained. $24 \mathrm{SRP}$ metabolic models via the inference of pan-genome analysis are constructed efficiently. The reference model of the well-studied model SRP Desulfovibrio vulgaris Hildenborough (DvH) is validated via Flux balance analysis (FBA). The DvH model predictions match reported experimental growth and energy yields, which demonstrates that the core metabolic model works successfully. Further, steady-state simulation of SRP metabolic models under different growth conditions shows how the use of different electron transfer pathways leads to energy generation. Three energy conservation mechanisms are identified, including menaquinone-based redox loop, hydrogen cycling, and proton pumping. Flavin-based electron bifurcation (FBEB) is also demonstrated to be an essential mechanism for supporting energy conservation. The developed models can be easily extended to other species of SRPs not examined in this study. More importantly, the present work develops an accurate and efficient approach for constructing metabolic models of multiple organisms, which can be applied to other critical microbes in environmental and industrial systems, thereby enabling the quantitative prediction of their metabolic behaviors to benefit relevant applications.
\end{abstract}

\section{Introduction}

Sulfate-reducing prokaryotes (SRPs) are anaerobic bacteria and archaea ubiquitously distributed in nature. They can couple dissimilatory sulfate reduction (DSR) to the oxidation of organic substrates or hydrogen/carbon dioxide in their energy metabolism, and therefore function as crucial mediators of the global sulfur and carbon cycle (Muyzer \& Stams, 2008; Rudolf K. Thauer et al., 2007). The corrosive and toxic hydrogen sulfide resulting from DSR is responsible for significant damage to the environment and industrial processes, and is also implicated in human health problems (Beech \& Sunner, 2007; Goldstein et al., 2003; Loubinoux et al., 2002; Pikaar et al., 2014; Rückert, 2016; Wang, 2012). On the other hand, the biogenically produced sulfide can be utilized beneficially to remove and recover heavy metals from groundwater and wastewater by capturing the dissolved metals into insoluble metal sulfides (Hao et al., 2014; K. Tang et al., 2009). In addition, the low redox potentials achieved by SRPs enable them to reduce several toxic metals and radionuclides, including divalent mercury $[\mathrm{Hg}(\mathrm{II})]$ and the oxyanions of chromium $[\mathrm{Cr}(\mathrm{VI})]$, uranium [U(VI)], and arsenic $[\mathrm{As}(\mathrm{V})]$ (Bruschi et al., 2007; Lloyd, 2003). SRPs exhibit versatile energy metabolic capacities 
by using various electron donors and carbon sources under a wide variety of environmental conditions, which also enables them to degrade municipal organic wastes, hydrocarbons, and crude oil in industrial and environmental applications (Hao et al., 2014; Ollivier et al., 2007; Rabus et al., 2013; Rabus et al., 2015). A fundamental understanding of the energy metabolism of SRPs growing with different energy sources is thus crucial for improving their performance in SRP-based biotechnologies, as well as for controlling their activity when they are undesired.

Extensive genomic and molecular studies of SRPs have been conducted in recent years, providing an enormous amount of information on their genetics, physiology, and biochemistry (Muyzer \& Stams, 2008; P. M. Pereira et al., 2008; Rabus et al., 2015; Walker et al., 2009). However, a systematic understanding of their energy metabolism remains elusive (Muyzer \& Stams, 2008; Rückert, 2016). For instance, DSR has long been recognized to be associated with energy conservation via oxidative phosphorylation, which implies an electron transport chain translocating charges across the cell membrane and generating a proton motive force (pmf) (Fitz \& Cypionka, 1989; Kobayashi et al., 1982; Peck, 1960; Wood, 1978). However, the terminal reductase of the DSR pathway, dissimilatory sulfite reductase (DsrAB), is located in the cytoplasm, thus failing to be directly involved in the membrane-associated charge translocation (I. A. C. Pereira et al., 2011; Rabus et al., 2015). The interaction of DsrAB with the membrane-bound electron transport chain to generate a pmf remains a mystery. More importantly, previous analyses indicated that the composition of energy metabolism proteins could vary significantly from one SRP to another (Rabus et al., 2015; Zhou et al., 2011), whereas current biochemical and molecular studies on SRPs are primarily focused on a few model organisms only, such as Desulfovibrio desulfuricans and Desulfovibrio vulgaris (Barton \& Fauque, 2009; Rabus et al., 2013). Besides, SRPs can switch from one mode of energy generation to another in response to shifting nutrient availability (P. M. Pereira et al., 2008; Walker et al., 2009). For these reasons, a systematic database describing the energy metabolic properties of SRPs is highly sought after.

Genome-scale metabolic models (GEMs) can systematically compile genomic, biochemical, genetic and highthroughput omics data, thereby forming a mathematically-structured knowledge-base for investigation of metabolic capabilities, generation and testing of hypotheses, and analysis of growth characteristics (King et al., 2016; Thiele \& Palsson, 2010). By using GEMs, not only engineers can obtain broader insights into the energy metabolism of SRPs, but also can quantitatively predict the metabolic behaviors of SRPs in engineered ecosystems. Despite their utility, high-quality GEM construction is a time-consuming and laborintensive process, involving multiple computational and experimental steps, which limits their application to only a few well-understood model organisms (J. Monk et al., 2014; Thiele \& Palsson, 2010). To address this challenge, automated GEM reconstruction tools have been developed that can generate an increasing number of GEMs (Gu et al., 2019; Machado et al., 2018). Nevertheless, without manual curation, the quality of an automatically generated draft GEM is always low, due to lower-confidence annotations, extensive gapfilling, and incompleteness and/or inaccuracy of the existing databases involving genes, enzymes, reactions, and metabolites (Edirisinghe et al., 2016; Gu et al., 2019). Many of these problems can be avoided by using a simplified core metabolic model comprised of only the well-studied and biologically critical pathways (Edirisinghe et al., 2016; J. D. Orth et al., 2010a), thus significantly simplifying the laborious construction process while maintaining the utility of GEMs. The core metabolic model aims at a focused understanding of central and energy metabolism. The chosen pathways in the model are often the subjects of textbooks and should be familiar to most readers with basic biochemistry knowledge. To further improve the accuracy and efficiency of metabolic model construction, comparative genomics has been integrated into GEMs by using curated reference GEMs from closely related organisms to derive multiple metabolic models for various strains of a single species (J. M. Monk et al., 2013) or multiple species of a single genus (Seif et al., 2018).

In view of the above, this study aims to develop an efficient approach for constructing metabolic models of multiple organisms, and to apply it to $24 \mathrm{SRP}$ species to study their energy metabolism quantitatively and systematically. To this end, a combination of comparative genomics via pan-genome analysis and the concept of core metabolic model has been applied. The global workflow illustrated in Figure 1 can be divided into four major steps: 1) pan-genome analysis of SRPs to obtain orthologous genes encoded proteins related to central and energy metabolism; 2) pairwise comparison of the gene-protein relations from pan-genome 
analysis and the reaction-protein relations from the model template to obtain gene-protein-reaction (GPR) associations for construction of $24 \mathrm{SRP}$ metabolic models; 3 ) validation of the metabolic model of the model SRP Desulfovibrio vulgarisHildenborough (DvH); and 4) Flux balance analysis (FBA) of the developed models to evaluate the energy metabolism of SRPs under different growth conditions.

\section{Model development}

\subsection{Pan-genome formulation}

Twenty-four completely assembled SRP genome sequences, including 5 archaea of the genus Archaeoglobus , 15 Gram-negative bacteria of the genus Desulfovibrio , and 4 Gram-positive bacteria of the genus Desulfotomaculum (Table 1), were extracted from the NCBI website (Brister et al., 2014). The genomic features, gene contents, and accession numbers of the genomic sequences of the SRPs are summarized in Table 1. Formulation of the pan-genome for each genus relies on iterative pairwise comparison of the selected genomes within the genus to identify groups of orthologous genes (OGs) that encode proteins with the same function. To improve the reliability of computation, orthologous groups were identified by a combination of two platforms: i) the EDGAR software platform which employs the BLAST Score Ratio Values (SRVs) with the orthology cutoff calculated from the analyzed genome set rather than using a fixed threshold (Blom et al., 2016), and ii) the pan-genome analysis pipeline BPGA with the default 50\% sequence identity as the cutoff value for ortholog clustering (Chaudhari et al., 2016). The final list of OGs (Table S1) comprised the OGs identified by both platforms. OGs predicted by either but not both of the platforms were manually inspected via BLASTP (protein-protein) search (Camacho et al., 2009). A phylogenetic tree to illustrate the genetic relationships between the $24 \mathrm{SRP}$ genomes was constructed using the FastTree tool (M. N. Price et al., 2010). Functional categories of genes within three different pan-genome subsets, i.e., core, accessory, and unique genomes, were predicted and classified using the Clusters of Orthologous Groups of proteins (COG) database (Tatusov et al., 2000). COG annotation was conducted on the WebMGA server, using the default e-value cutoff of 0.001 for prediction (Wu et al., 2011).

\subsection{Construction of multiple metabolic models}

The concept of core metabolic model was adopted in this study to focus on energy metabolism and improve the efficiency of model construction. The model construction workflow began with creating a core model template (Figure 1). The SRP core model template consisted of glycolysis, tricarboxylic acid cycle (TCA) cycle, pentose phosphate pathway, fermentation, DSR, and various electron transport chain (ETC) pathways. Core reactions with associated functional proteins in the model template were derived from Edirisinghe et al. (2016) and J. D. Orth et al. (2010a) in the context of pan-genome analysis. In addition, DSR and ETC pathways were included through literature mining and curation.

The ortholog table obtained from pan-genome analysis, which consisted of orthologous genes associated with functional proteins, was compared against the SRP core model template, which encompassed functional proteins associated with biochemical reactions. This pairwise comparison was to ascertain the presence or absence of specific biochemical reactions and pathways, resulting in a set of GPR associations. Once the GPR associations were determined, the related biochemistry data was propagated to construct a draft metabolic model. An objective function (OF) of biomass biosynthesis, which was optimized during FBA to predict flux profiles (Schuetz et al., 2007), was obtained from Edirisinghe et al. (2016) and added to each draft model. The draft model was further imported into the Cobra toolbox in MATLAB (Heirendt et al., 2017) to conduct minimum gap-filling. A total of 24 metabolic models of SRPs were constructed, among which, DvH is chosen as the model organism for model validation, because it is well studied and encompasses versatile ETC pathways that are shared by most SRP species (I. A. C. Pereira et al., 2011). Both growth associated maintenance (GAM) and non-growth associated maintenance (NGAM) are added to the DvH model. NGAM quantifies the energy required by SRPs to maintain themselves in a given environment while GAM quantifies 
growth energy requirements not included in the metabolic model (Kavvas et al., 2018). Literature data obtained from chemostat experiments are used for GAM and NGAM calculation (Badziong \& Thauer, 1978; Traore et al., 1981).

\subsection{Model simulations by flux balance analysis}

To make rigorous and quantitative growth predictions, metabolic models were transferred into stoichiometric matrix $(S)$, in which $S_{i j}$ represented the stoichiometric coefficient of metabolite $i$ in reaction $j$. The vector of reaction fluxes (reaction rates) is represented by a vector $v$. FBA was used to optimize specific OFs such as biomass or targeted metabolites under steady-state criteria $(S \cdot v=0)$, thereby making it possible to predict the growth rate of an organism or the rate of production of a biotechnologically important metabolite (Oberhardt et al., 2009; Jeffrey D Orth et al., 2010b; N. D. Price et al., 2004). In this study, simulations are performed by assuming maximal biomass production to be the OF of SRPs. Limiting substrate uptake rates were adopted from literature data (Badziong \& Thauer, 1978; Traore et al., 1981). Predicted flux of the simulation could be visualized in an Escher map (King et al., 2015).

\section{Results and discussion}

\subsection{Characteristics of the SRP core genome and pan-genome}

The pan-genomes of three SRP genera are assembled to highlight the genetic and metabolic diversity of SRPs. Based on the sequence similarity analysis, a phylogenetic tree created for all 24 SRPs together shows a clear separation of Archaeoglobus ,Desulfovibrio , and Desulfotomaculum (Figure S1), demonstrating that they belong justifiably to three different genera. Pan-genome of every genus can be subdivided into three subsets: (i) the core genome (genes shared among all species and mostly play a housekeeping role), (ii) the accessory genome (genes present in some but not all species and contribute to species adaptation to different conditions), and (iii) the unique genome (species-specific genes). Pan-genome subset distribution and function profile of Archaeoglobus, Desulfovibrio , and Desulfotomaculumare shown in Figure 2, and pangenome and core genome development plot projections are provided in supplementary Figure S2. The core genomes of all three genera display a similar evolution, which rapidly stagnates at close to 800 genes. The proportion of core genome in the pan-genome varies significantly (from 3.7\% to 25.8\%) due to the differences in pangenome size, but remains relatively low, revealing a rather high inter-species diversity of each genus. In terms of functional annotation according to COGs, almost half of the genes from the core genomes of the three genera are devoted to translation, energy metabolism, and the metabolism of building blocks (carbohydrates, amino acids, nucleotides, coenzymes, and lipids) (Figure 2), which supports Ouzounis and Kyrpides (1996)'s hypothesis that metabolism and translation are conserved and close to the last universal ancestor. Besides, a significant fraction (more than 20\%) of core genes of Archaeoglobus andDesulfotomaculum encodes poorly characterized proteins (categories R and S) whose function is hypothetical, while that fraction of Desulfovibrio is only $8.2 \%$ because Desulfovibriostrains have been intensively studied (Barton \& Fauque, 2009).

Key genes and associated functional proteins involved in central carbon and energy metabolism are provided in the ortholog table generated from the pan-genome analysis (Table S1). Overall, all SRP species contain complete gene sets for glycolysis, while adopting an incomplete pentose phosphate pathway and an incomplete TCA cycle. A complete set of genes for the Wood-Ljungdahl pathway (Schauder et al., 1986; Spormann \& Thauer, 1988) was identified in Archaeoglobus. The essential enzymes for DSR are conserved in all studied SRPs, including sulfate transporters, ATP sulfurylase (Sat), APS reductase (AprAB), and dissimilatory sulfite reductase (DsrAB). Two electron-transporting enzyme complexes, dissimilatory sulfite reductase (DsrMKJOP) and quinone-interacting membrane-bound oxidoreductase (QmoABC), are conserved across SRPs, which are assumed to be involved in electron transfer from electron donors to the terminal reductases AprAB and DsrAB respectively. In addition,Desulfovibrio contains several other electron transfer 
complexes such as Hmc (high-molecular-weight complex), Tmc (transmembrane complex), Rnf (ferredoxin: NAD reductase), Nuo (NADH: quinone oxidoreductase complex), and energy-conserving hydrogenases (Ech or Coo), but few of them are found in Archaeoglobus or Desulfotomaculum, suggesting that electron transfer for sulfate reduction is substantially different among SRPs.

\subsection{Model construction and validation}

Based on the orthologous relationships resulted from pan-genome analysis, core metabolic models of the 24 SRPs are constructed and used to simulate their metabolic capabilities and growth characteristics under different growth conditions. The resulting core metabolic models consist of 91-110 reactions (Figure 3). They share most of the reactions related to central carbon metabolism but vary significantly in electron transfer pathways. Clustering of the models shows that the phylogenetically closely related SRP species have similar metabolic capabilities. Detailed information of all the reactions and the GPR associations, as well as the model files of 24 SRPs can be found on Github (https://github.com/TANG-Wentao/SRP_MetabolicModel).

To demonstrate the predictive accuracy of the core metabolic model, steady-state growth simulation was performed on the metabolic model under two different growth conditions: lactate-limited and hydrogenlimited sulfate respiration (LS and HS, respectively). The predicted growth yields ( $\left.\mathrm{Y}_{\mathrm{X} / \mathrm{S}}\right)$ and specific growth rates $(\mu)$ are compared to the experimentally determined values. Multiple literature datasets describing growth yields of $\mathrm{DvH}$ are available. The growth yields are dependent on the specific growth rate $(\mu)$ and the maintenance coefficient (m) (Russell \& Cook, 1995); thus, growth rates and growth yields from both experimental results and model predictions are compiled according to Eq. 1 (Pirt, 1965). As shown in Figure 4, under LS and HS conditions, the predicted growth yields and specific growth rates of DvH are in close agreement with the experimental data.

$$
\frac{1}{Y}=\frac{m}{\mu}+\frac{1}{Y_{\max }}
$$

For HS growth, a net synthesis of 1.21 ATP molecule per sulfate reduced is predicted from metabolic modeling, which is close to the theoretical value proposed by a previous study (Badziong \& Thauer, 1978). By comparing the growth yield data and the growth energy requirement of $D$. vulgaris under HS growth, a net yield of 1 ATP molecule per sulfate reduced was proposed by Badziong and Thauer (1978). The stoichiometry of cell synthesis and energy reactions for growth ofDesulfovibrio under LS growth is provided in Table 2. More than $90 \%$ of electron donor is used for energy generation, and the rest is used for cell synthesis, which is in agreement with previous studies (Noguera et al., 1998; Traore et al., 1981). Our results demonstrate that the high-quality core metabolic model equipped with improved annotations in ETC pathways can accurately predict the growth characteristics and energy yield of SRPs, providing a basis for further quantitative analysis of energy metabolism.

\subsection{Lactate-sulfate (LS) respiration}

Lactate is the most widely used growth substrate by known SRPs and often applied for cultivation purposes. The central carbon metabolism and the predicted metabolic flux of essential reactions associated with energy generation under LS growth are shown in Figure 5 and Figure 6a. Lactate oxidation to acetate is mediated by lactate dehydrogenase (Ldh), and the produced pyruvate from lactate oxidation is then converted to acetyl$\mathrm{CoA}$ via pyruvate: formate lyase (Pfl) and/or pyruvate: ferredoxin oxidoreductase (Por). The predicted pathway of lactate oxidation agrees well with previous studies (Keller \& Wall, 2011; P. M. Pereira et al., 2008). Acetyl-CoA is mainly used to produce ATP through substrate-level phosphorylation by Acetyl-CoA synthetase (ADP-forming, Acs), or phosphotransacetylase (Pta)/acetate kinase (Ack) couple. Consistent with previous genome analysis, this pathway is highly conserved in SRPs (Vita et al., 2015). For SRPs without Acs or Pta, such as Desulfovibrio magneticus andDesulfovibrio gigas, acetyl-CoA is predicted to 
be converted to acetaldehyde by aldehyde:ferredoxin oxidoreductase (Aor) or to citrate by citrate synthase (CIT), which are in turn converted to ethanol and succinate respectively.

For Desulfovibrio and Desulfotomaculum species except forD.magneticus and D. gigas, a theoretical ratio of acetate excretion to lactate utilization of 1:1 is expected in energy metabolism based on the stoichiometry of this pathway, and an average acetate-to-lactate ratio of 0.907:1 is predicted (Figure 6b \& Table 2). A difference exists between the theoretical ratio and the predicted value because a small amount of acetyl-CoA is used for the synthesis of the building blocks, and this part of acetyl-CoA plays a non-energetic, assimilatory role in SRPs. For the complete oxidizers, e.g.Archaeoglobus species, acetyl-CoA is predicted to be further utilized by the Wood-Ljungdahl pathway (Schauder et al., 1986; Spormann \& Thauer, 1988) for energy generation by coupling the endergonic oxidation of acetate to $\mathrm{CO}_{2}$, thus no acetate is excreted and the ratio of $\mathrm{CO}_{2}$ to lactate is triple that of complete oxidizers (Figure $6 \mathrm{~b}$ ). The ATP yield (ATP/sulfate ratio) varies significantly across different SRPs, with a predicted average of 2.35 .

Electrons generated through lactate oxidation are assumed to be used indirectly for sulfate reduction by the hydrogen $\left(\mathrm{H}_{2}\right)$ cycling pathway (Odom \& Jr, 1981). Another study (Lupton et al., 1984) argued that $\mathrm{H}_{2}$ is not an obligatory intermediate, but is produced only as a mechanism to control the redox state of internal electron carriers, and the electrons transport directly through membrane-bound electron carriers to sulfate. Experimental evidence partially supports each hypothesis (Keller \& Wall, 2011; Noguera et al., 1998). Based on our modeling work (Figure 6a and Figure 7), these two electron transport mechanisms can complementrather than compete with - each other for Desulfovibrio. As shown in Figure 7, for the direct pathway, electrons flow from lactate to the membrane-bound electron carrier menaquinone (MQ) and then transfer to sulfate reduction (via Qmo and DsrMK) without $\mathrm{H}_{2}$ involvement. In general, the consumption and evolution of $\mathrm{H}_{2}$ are mediated by hydrogenases (Hase), which are redox metalloenzymes that catalyze the reversible oxidation of $\mathrm{H}_{2}$ (Vignais \& Colbeau, 2004). For the $\mathrm{H}_{2}$ cycling pathway, electron equivalents $\left(\mathrm{Fd}_{\mathrm{red}}\right.$ and $\mathrm{MQH}_{2}$ ) and protons generated from Ldh will react with membrane-bound Hase Ech and/or Coo to form $\mathrm{H}_{2}$, which will diffuse to the periplasm and be oxidized back to protons and electrons by periplasmic Hase (Hase_p). The electrons from $\mathrm{H}_{2}$ oxidation are transported to the electron equivalent Type I cytochrome $\mathrm{c}_{3}\left(\mathrm{TpI}_{3}\right)$, and then transferred back to the cytoplasm via Hmc and Qrc for sulfate reduction, creating a transmembrane proton gradient for ATP synthesis. This predicted phenomenon is supported by a previous study of Desulfovibrio gigas, where only one periplasmic and one cytoplasmic Hase are present. Single deletion of mutants for each of these proteins showed slightly lower growth rates with lactate-sulfate or pyruvate-sulfate than the wild-type strain, suggesting that $\mathrm{H}_{2}$ cycling can be compensated by the direct MQ mediated electron transport (Morais-Silva et al., 2013). Based on our simulation, the so-called $\mathrm{H}_{2}$-cycling mechanism is responsible for around half of the electron transfer inDesulfovibrio under LS growth, except Desulfovibrio alaskensis G20 who contains no Ech or Coo.

Genera Archaeoglobus and Desulfotomaculum do not use the $\mathrm{H}_{2}$ cycling pathway for energy conservation under LS growth due to the lack of a periplasmic space for $\mathrm{H}_{2}$ oxidation and the absence of transmembrane electron transfer proteins such as Qrc and Hmc. For Desulfotomaculum species, $\mathrm{H}_{2}$ generated from Ldh is predicted to involve in the flavin-based electron bifurcation mechanism (see section 3.5 for details) for the generation of reduced ferredoxin $\left(\mathrm{Fd}_{\text {red }}\right)$ and $\mathrm{NADH}$ via cytoplasmic Hase (Hase_c), thus serving as a redox balancing strategy as suggested by Lupton et al. (1984). NADH and $\mathrm{Fd}_{\text {red }}$ further drive sulfite reduction via heterodisulfide reductase (Hdr) and/or dissimilatory sulfite reductase (DsrC). For Archaeoglobus species, $\mathrm{H}_{2}$ generation is not predicted during LS growth, and a new kind of electron equivalent, reduced coenzyme $\mathrm{F}_{420}\left(\mathrm{~F}_{420} \mathrm{H}_{2}\right)$, is introduced. $\mathrm{F}_{420} \mathrm{H}_{2}$ is generated as a result of complete oxidation of lactate into $\mathrm{CO}_{2}$ via the Wood-Ljungdahl pathway, and is further converted to $\mathrm{MQH}_{2}$ by the membrane-bound electron carriers Fqo $\left(\mathrm{F}_{420} \mathrm{H}_{2}\right.$ : quinone oxidoreductase). NADH is somewhat a substitute for $\mathrm{H}_{2}$ in Archaeoglobusand Desulfotomaculum. It not only works as the redox currency, but also directly drives energy conservation by Nuo (NADH: quinone oxidoreductase). 


\subsection{Hydrogen-sulfate (HS) respiration}

Molecular hydrogen $\left(\mathrm{H}_{2}\right)$, an electron sink product of anaerobic fermentation, can be utilized via DSR, methanogenesis, and acetogenesis to enable more efficient energy recovery from organic substrates (Rowland et al., 2018). When $\mathrm{H}_{2}$ is the only available electron donor, most SRPs will grow heterotrophically on acetate, and carbon dioxide can serve as a supplementary carbon source. The average carbon dioxide-toacetate ratio of SRPs is predicted to be 0.96 , which means $67.6 \%$ of the carbon is derived from acetate and the other $32.4 \%$ comes from carbon dioxide (Figure 8 \& Table 2). This result agrees well with experimental findings from previous work (Badziong \& Thauer, 1978), which reported that $70 \%$ of the carbon is derived from acetate, and the remaining $30 \%$ comes from carbon dioxide.Archaeoglobus sulfaticallidus are capable of coupling chemolithoautotrophic growth on $\mathrm{H}_{2} / \mathrm{CO}_{2}$ to sulfate reduction in addition to heterotrophic growth. For Archaeoglobus other than A. sulfaticallidus, although the machinery for $\mathrm{CO}_{2}$ fixation via the WoodLjungdahl pathway exists, autotrophic growth with $\mathrm{H}_{2} / \mathrm{CO}_{2}$ is not feasible. This is because $\mathrm{Fd}_{\text {red }}$ generated from Hase is completely used up for APS reduction (Qmo), $\mathrm{CO}_{2}$ fixation through the Wood-Ljungdahl pathway cannot be promoted without $\mathrm{Fd}_{\text {red }}$.

Metabolic fluxes predicted by FBA for essential reactions related to central carbon metabolism and energy generation are shown in Figure 8a. As expected, a strikingly different flux distribution is observed under HS growth condition as compared to LS growth condition. With $\mathrm{H}_{2}$ serving as the electron donor for growth,Desulfovibrio is predicted to establish a pmf directly by periplasmic $\mathrm{H}_{2}$ oxidation (Hase_p). Besides, the membrane-bound Hase Ech can function in reverse to oxidize $\mathrm{H}_{2}$ and generate $\mathrm{Fd}_{\text {red }}$ for carbon fixation during growth with $\mathrm{H}_{2}$ for severalDesulfovibrio species. Archaeoglobus andDesulfotomaculum species are predicted to use soluble cytoplasmic Hases ( $\mathrm{MvH}$ and Hase_c) that are not present in Desulfovibrio , and these Hases are involved in electron bifurcation mechanisms to produce $\mathrm{Fd}_{\mathrm{red}}$ and balance the redox state of internal electron carriers (see 3.5 for details). Por (pyruvate: ferredoxin oxidoreductase) involves in energy metabolism of all examined SRPs under both LS and HS growth. It can work bidirectionally to oxidize pyruvate into acetyl-CoA and $\mathrm{CO}_{2}$ accompanied with $\mathrm{Fd}_{\text {red }}$ generation under LS growth, or to synthesize pyruvate via $\mathrm{CO}_{2}$ fixation fueled by $\mathrm{Fd}_{\text {red }}$ under $\mathrm{HS}$ growth. For several SRPs (Figure 8a), formate is predicted to be generated from pyruvate via $\mathrm{Pfl}$ and is then oxidized to protons and electrons by periplasmic formate dehydrogenase $(\mathrm{Fdh})$. This formate cycling pathway performs a similar function to the $\mathrm{H}_{2}$ cycling pathway, which is supported by the transcriptome study of P. M. Pereira et al. (2008).

\subsection{Energy conservation mechanisms}

In sulfate respiration, energy is mainly generated through the conversion of the free energy of a redox reaction into a pmf, which drives ATP production by ATP synthase (ATPase). Despite intensive study, the mechanism of energy conservation during sulfate respiration is still incompletely understood. By simulating the steady-state growth of 24 SRPs under different growth conditions, three mechanisms are proposed for energy conservation via oxidative phosphorylation in this study, as shown in Figure 9.

The MQ-based redox loop is the universal machine used for energy conservation in SRPs. In this scenario, proton translocation is achieved via charge separation performed by coupling MQ reduction with $\mathrm{MQH}_{2}$ oxidation (Simon et al., 2008). A MQ reductase such as Qrc, Hmc, Nuo, and Fqo, together with a $\mathrm{MQH}_{2}$ oxidase such as Qmo, Coo, and DsrMK, constitute the two arms of an energy-conserving redox loop (Figure 9a). The charge separation is established by electron extraction from the positive $(\mathrm{P})$ side and proton uptake on the negative $(\mathrm{N})$ side of the membrane by MQ reductases, paired with electron transfer to the $\mathrm{N}$ side and proton release to the $\mathrm{P}$ side by $\mathrm{MQH}_{2}$ oxidases (Figure 9a). The second energy conservation mechanism is to build up a pmf via direct proton pumping (Figure 9b). Several electron transfer complexes capable of proton pumping are found in SRPs, including Nuo, Fqo, Rnf, Ech, Coo, and PPi (inorganic pyrophosphate), which provides SRPs with great flexibility in terms of energy metabolism. The Ech and Coo Hases are involved in both direct proton pumping and indirect $\mathrm{H}_{2}$ cycling (Figure 9c). Ech and Coo can convert protons and electron equivalents $\left(\mathrm{Fd}_{\text {red }}\right.$ and $\left.\mathrm{MQH}_{2}\right)$ that are generated by the oxidation of organic compounds (e.g., lactate) to $\mathrm{H}_{2}$, simultaneously translocate protons. The $\mathrm{H}_{2}$ is then reoxidized to protons and electrons ( $\mathrm{TpIc}_{3}$ 
pool) by periplasmic Hase (Hase_p). The $\mathrm{H}_{2}$ cycling has to be operated at very low $\mathrm{H}_{2}$ partial pressure to allow $\mathrm{H}_{2}$ generation through Ech and Coo thermodynamically feasible. This is because the $\mathrm{H}^{-} / \mathrm{H}_{2}$ couple has lower redox potential $\left(\mathrm{E}^{\circ}=-414 \mathrm{mV}\right)$ than $\mathrm{MQ} / \mathrm{MQH}_{2}\left(\mathrm{E}^{\circ}=-74 \mathrm{mV}\right)$ and $\mathrm{Fd}_{\mathrm{red}} / \mathrm{Fd}_{\mathrm{ox}}\left(\mathrm{E}^{\circ}=-398 \mathrm{mV}\right)$ at standard conditions (Table 3), and the decrease of $\mathrm{H}_{2}$ partial pressure to $1 \mathrm{~Pa}$ will increase the lower redox potential of $\mathrm{H}^{-} / \mathrm{H}_{2}$ couple to $-270 \mathrm{mV}$. Formate cycling across the membrane works as an alternative energetic pathway of $\mathrm{H}_{2}$ cycling, and carbon monoxide $(\mathrm{CO})$ cycling is also proposed during fermentative growth (Voordouw, 2002).

Flavin-based electron bifurcation (FBEB) is a novel mechanism by which a hydride electron pair from $\mathrm{H}_{2}$, $\mathrm{NAD}(\mathrm{P}) \mathrm{H}, \mathrm{F}_{420} \mathrm{H}_{2}$, or formate is split by flavoproteins into one-electron with lower redox potential and one with a higher redox potential than that of the electron pair (Buckel \& Thauer, 2018). FBEB is not directly involved in energy conservation; instead, it supports energy conservation through Qmo, Rnf, Coo, and Ech by generating $\mathrm{Fd}_{\text {red }}$ from relatively high-potential electron donors such as NADH (Figure 9d). The discovery of electron bifurcation also helps to resolve the physiological role of MQ in DSR. MQ pool is widely accepted as a critical mediator in the electron transfer and energy generation in SRPs. However, the redox potential of $\mathrm{MQ} / \mathrm{MQH}_{2}\left(\mathrm{E}^{\circ}=-74 \mathrm{mV}\right)$ is higher than most of the redox couples found in SRPs (Table 3) and is very similar to APS $/ \mathrm{HSO}_{3}{ }^{-}\left(\mathrm{E}^{\circ}=-65 \mathrm{mV}\right)$. Therefore, $\mathrm{MQ}$ can readily receive electrons from other electron donors, whereas donating electrons from $\mathrm{MQH}_{2}$ to other electron acceptors seems thermodynamically unfavorable. Via electron bifurcation, $\mathrm{MQH}_{2}$ oxidation can be achieved by introducing a second electron donor (e.g., $\mathrm{Fd}_{\mathrm{red}}$ ) with lower redox potential, so that electron transfer via Qmo and Coo can proceed (Figure 9 a and c).

Overall, MQ-based redox loop and FBEB are widely used to conserve energy in SRPs. $\mathrm{H}_{2}$ cycling is restricted toDesulfovibrio , and Nuo induced proton pumping plays a crucial role in Archaeoglobus and Desulfotomaculum . A wide variety of proteins are involved in energy conservation of SRPs, and our simulations show that alternative energy-conserving proteins and different energy-conserving mechanisms can be used in response to shifting nutrient availability, which enables SRPs to thrive in a range of different environmental conditions.

\section{Conclusion}

In this study, we developed a semi-automatic approach for accurate and efficient construction of metabolic models of multiple SRP species by applying pan-genome analysis and the concept of core metabolic model. The constructed core metabolic model for $\mathrm{DvH}$ can successfully predict growth yield and several growth characteristics. Flux balance analysis of the SRP core metabolic models under different respiratory conditions has given birth to an overall energy conservation model. In addition to the previously reported hydrogen cycling model, the functions of various membrane-bound electron transfer complexes in energy conservation were elucidated, the recently proposed FBEB mechanism for energy metabolism was also supported in this study. Such information is essential for understanding the versatile metabolic capacities of SRPs and for developing SRP-based biotechnologies. More importantly, the present work developed an efficient approach for construction of multiple metabolic models, which creates an opportunity to explore and manipulate the metabolism of key microbes in the environment and biotechnology.

\section{Acknowledgments}

This research was supported by the Key Program of National Natural Science of China (grant no. 51638005), the Hong Kong Innovation and Technology Commission (grant no. ITC-CNERC14EG03), the National Natural Science Foundation of China (grant no. 51778165) and the Science and Technology Development Fund, Macau SAR (grant nos. 0040/2018/A1 and 0104/2018/A3). 


\section{Supplementary materials}

Model files of 24 SRP species in SBML format as well as other supplementary materials are publically available at Github (https://github.com/TANG-Wentao/SRP_MetabolicModel).

\section{References}

Badziong, W., \& Thauer, R. K. (1978). Growth yields and growth rates of Desulfovibrio vulgaris (Marburg) growing on hydrogen plus sulfate and hydrogen plus thiosulfate as the sole energy sources. Archives of Microbiology, 117 (2), 209-214.

Barton, L. L., \& Fauque, G. D. (2009). Chapter 2 Biochemistry, Physiology and Biotechnology of SulfateReducing Bacteria.Advances in Applied Microbiology, 68 (09), 41-98. doi:10.1016/S0065-2164(09)01202-7

Beech, I. B., \& Sunner, J. (2007). Sulphate-reducing bacteria and their role in corrosion of ferrous materials. In Sulphate-reducing bacteria: environmental and engineered systems (pp. 459-482): Cambridge University Press.

Blom, J., Kreis, J., Spänig, S., Juhre, T., Bertelli, C., Ernst, C., \& Goesmann, A. (2016). EDGAR 2.0: an enhanced software platform for comparative gene content analyses. Nucleic acids research, 44 (W1), W28-W28. doi:10.1093/nar/gkw255

Brister, J. R., Ako-Adjei, D., Bao, Y., \& Blinkova, O. (2014). NCBI viral genomes resource. Nucleic acids research, 43 (D1), D577-D577. doi:10.1093/nar/gku1207

Bruschi, M., Barton, L. L., Goulhen, F., \& Plunkett, R. M. (2007). Enzymatic and genomic studies on the reduction of mercury and selected metallic oxyanions by sulphate-reducing bacteria. InSulphate-Reducing Bacteria: Environmental and Engineered Systems(pp. 435-457): Cambridge University Press.

Buckel, W., \& Thauer, R. K. (2018). Flavin-based electron bifurcation, ferredoxin, flavodoxin, and anaerobic respiration with protons $(\mathrm{Ech})$ or $\mathrm{NAD}+(\mathrm{Rnf})$ as electron acceptors: A historical review. Frontiers in microbiology, 9 , 401.

Camacho, C., Coulouris, G., Avagyan, V., Ma, N., Papadopoulos, J., Bealer, K., \& Madden, T. L. (2009). BLAST+: architecture and applications. BMC Bioinformatics, 10 (1), 421-421. doi:10.1186/1471-2105-10421

Chaudhari, N. M., Gupta, V. K., \& Dutta, C. (2016). BPGA-an ultra-fast pan-genome analysis pipeline. Scientific reports, 6 , 24373-24373. doi:10.1038/srep24373

Edirisinghe, J. N., Weisenhorn, P., Conrad, N., Xia, F., Overbeek, R., Stevens, R. L., \& Henry, C. S. (2016). Modeling central metabolism and energy biosynthesis across microbial life. BMC Genomics, 17 (1), 568-568. doi:10.1186/s12864-016-2887-8

Fitz, R. M., \& Cypionka, H. (1989). A study on electron transport-driven proton translocation in Desulfovibrio desulfuricans. Archives of Microbiology, 152 (4), 369-376. doi:10.1007/BF00425175

Goldstein, E. J., Citron, D. M., Peraino, V. A., \& Cross, S. A. (2003). Desulfovibrio desulfuricans bacteremia and review of human Desulfovibrio infections. Journal of clinical microbiology, 41 (6), 2752-2754. doi:10.1128/jcm.41.6.2752-2754.2003

Gu, C., Kim, G. B., Kim, W. J., Kim, H. U., \& Lee, S. Y. (2019). Current status and applications of genome-scale metabolic models. Genome Biology, 20 (1), 121. doi:10.1186/s13059-019-1730-3

Hao, T., Xiang, P., Mackey, H. R., Chi, K., Lu, H., Chui, H., . . Chen, G. (2014). A review of biological sulfate conversions in wastewater treatment. Water research, $65,1-21$. doi:10.1016/j.watres.2014.06.043 
Heirendt, L., Arreckx, S., Pfau, T., Mendoza, S. N., Richelle, A., Heinken, A., . . Wachowiak, J. (2017). Creation and analysis of biochemical constraint-based models: the COBRA Toolbox v3. 0.arXiv preprint arXiv:1710.04038 .

Kavvas, E. S., Seif, Y., Yurkovich, J. T., Norsigian, C., Poudel, S., Greenwald, W. W., . . Monk, J. M. (2018). Updated and standardized genome-scale reconstruction of Mycobacterium tuberculosis H37Rv, iEK1011, simulates flux states indicative of physiological conditions.BMC systems biology, 12 (1), 25-25. doi:10.1186/s12918-018-0557-y

Keller, K. L., \& Wall, J. D. (2011). Genetics and molecular biology of the electron flow for sulfate respiration in Desulfovibrio.Frontiers in microbiology, 2 , 135-135. doi:10.3389/fmicb.2011.00135

King, Z. A., Lu, J., Dräger, A., Miller, P., Federowicz, S., Lerman, J. A., . . Lewis, N. E. (2016). BiGG Models: A platform for integrating, standardizing and sharing genome-scale models. Nucleic Acids Research . doi:10.1093/nar/gkv1049

Kobayashi, K., Hasegawa, H., Takagi, M., \& Ishimoto, M. (1982). Proton translocation associated with sulfite reduction in a sulfate-reducing bacterium, Desulfovibrio vulgaris. FEBS Letters, 142 (2), 235-237. doi:10.1016/0014-5793(82)80142-7

Lloyd, J. R. (2003). Microbial reduction of metals and radionuclides.FEMS microbiology reviews, 27 (2-3), 411-425. doi:10.1016/S0168-6445(03)00044-5

Loubinoux, J., Bronowicki, J.-P., Pereira, I. A., Mougenel, J.-L., \& Le Faou, A. E. (2002). Sulfate-reducing bacteria in human feces and their association with inflammatory bowel diseases. FEMS microbiology ecology, 40 (2), 107-112. doi:10.1016/S0168-6496(02)00201-5

Lundblad, R. L., \& Macdonald, F. (2018). Handbook of biochemistry and molecular biology (4 ed.): CRC Press.

Lupton, F. S., Conrad, R., \& Zeikus, J. G. (1984). Physiological function of hydrogen metabolism during growth of sulfidogenic bacteria on organic substrates. Journal of Bacteriology, 159 (3), 843-849.

Machado, D., Andrejev, S., Tramontano, M., \& Patil, K. R. (2018). Fast automated reconstruction of genomescale metabolic models for microbial species and communities. Nucleic Acids Research, 46 (15), 7542-7553. doi:10.1093/nar/gky537

Monk, J., Nogales, J., \& Palsson, B. O. (2014). Optimizing genome-scale network reconstructions. Nature Biotechnology, 32 (5), 447-447. doi:10.1038/nbt.2870

Monk, J. M., Charusanti, P., Aziz, R. K., Lerman, J. A., Premyodhin, N., Orth, J. D., . . Palsson, B. Ø. (2013). Genome-scale metabolic reconstructions of multiple Escherichia coli strains highlight strain-specific adaptations to nutritional environments.Proceedings of the National Academy of Sciences, 110 (50), 2033820343. doi:10.1073/pnas. 1307797110

Morais-Silva, F. O., Santos, C. I., Rodrigues, R., Pereira, I. A. C., \& Rodrigues-Pousada, C. (2013). Role of $\mathrm{Hyn} \mathrm{AB}$ and Ech, the only two hydrogenases found in the model sulfate reducer Desulfovibrio gigas. Journal of Bacteriology, 195 (20), 13-13. doi:10.1128/Jb.00411-13

Muyzer, G., \& Stams, A. J. M. (2008). The ecology and biotechnology of sulphate-reducing bacteria. Nature Reviews Microbiology, 6 (6), 441-454. doi:10.1038/nrmicro1892

Nethe-Jaenchen, R., \& Thauer, R. K. (1984). Growth Yields and Saturation Constant of DesulfovibrioVulgaris in Chemostat Culture.Archives of Microbiology, 137 (3), 236-240. doi:Doi 10.1007/Bf00414550

Noguera, D. R., Brusseau, G. A., Rittmann, B. E., \& Stahl, D. A. (1998). Christos. Biotechnology and bioengineering, 59 (6), 732-746. doi:10.1002/(sici)1097-0290(19980920)59:6<732::aid-bit10>3.0.co;2-7 
Oberhardt, M. A., Palsson, B., \& Papin, J. A. (2009). Applications of genome-scale metabolic reconstructions. Molecular systems biology, 5 (1), 320. doi:10.1038/msb.2009.77

Odom, J. M., \& Jr, H. D. P. (1981). Hydrogen cycling as a general mechanism for energy coupling in the sulfate-reducing bacteria, Desulfovibrio sp. FEMS microbiology letters, 12 (1), 47-50. doi:10.1111/j.15746968.1981.tb07609.x

Ollivier, B., Cayol, J.-L., \& Fauque, G. (2007). Sulphate-reducing bacteria from oil fields environments and deep-sea hydrothermal vents. In Sulphate-Reducing Bacteria: Environmental and Engineered Systems (pp. 305-328): Cambridge University Press.

Orth, J. D., Fleming, R. M., \& Palsson, B. (2010a). Reconstruction and Use of Microbial Metabolic Networks: the Core Escherichia coli Metabolic Model as an Educational Guide. EcoSal Plus, 4 (1). doi:10.1128/ecosalplus.10.2.1

Orth, J. D., Thiele, I., \& Palsson, B. O. (2010b). What is flux balance analysis? Nature biotechnology, 28 (3), 245-248.

Ouzounis, C., \& Kyrpides, N. (1996). The emergence of major cellular processes in evolution. FEBS letters, 390 (2), 119-123. doi:10.1016/0014-5793(96)00631-x

Pankhania, I. P., Gow, L. A., \& Hamilton, W. A. (1986). The Effect of Hydrogen on the Growth of Desulfovibrio-Vulgaris (Hildenborough) on Lactate. Journal of General Microbiology, 132 , 3349-3356.

Peck, H. D. (1960). Evidence for oxidative phosphorylation during the reduction of sulfate with hydrogen by Desulfovibrio desulfuricans. The Journal of biological chemistry, 235 (9), 2734-2738.

Pereira, I. A. C., Ramos, A., Grein, F., Marques, M., Da Silva, S., \& Venceslau, S. (2011). A Comparative Genomic Analysis of Energy Metabolism in Sulfate Reducing Bacteria and Archaea. Frontiers in Microbiology, 2 (69). doi:10.3389/fmicb.2011.00069

Pereira, P. M., He, Q., Valente, F. M. A., Xavier, A. V., Zhou, J., Pereira, I. A. C., \& Louro, R. O. (2008). Energy metabolism in Desulfovibrio vulgaris Hildenborough: Insights from transcriptome analysis. Antonie van Leeuwenhoek, 93 (4), 347-362. doi:10.1007/s10482-007-9212-0

Pikaar, I., Sharma, K. R., Hu, S., Gernjak, W., Keller, J., \& Yuan, Z. (2014). Reducing sewer corrosion through integrated urban water management. Science, 345 (6198), 812-814. doi:10.1126/science.1251418

Price, M. N., Dehal, P. S., \& Arkin, A. P. (2010). FastTree 2-Approximately Maximum-Likelihood Trees for Large Alignments.PloS one, 5 (3), e9490. doi:10.1371/journal.pone.0009490

Price, N. D., Reed, J. L., \& Palsson, B. O. (2004). Genome-scale models of microbial cells: evaluating the consequences of constraints. Nature Reviews Microbiology, 2 (11), 886-886. doi:10.1038/nrmicro1023

Rabus, R., Hansen, T. A., \& Widdel, F. (2013). Dissimilatory sulfate- and sulfur-reducing prokaryotes. In The prokaryotes: prokaryotic physiology and biochemistry (4 ed., pp. 309-404): Springer Berlin Heidelberg.

Rabus, R., Venceslau, S. S., Woehlbrand, L., Voordouw, G., Wall, J. D., \& Pereira, I. A. (2015). A postgenomic view of the ecophysiology, catabolism and biotechnological relevance of sulphate-reducing prokaryotes. In Advances in microbial physiology (Vol. 66, pp. 55-321): Elsevier.

Rowland, I., Gibson, G., Heinken, A., Scott, K., Swann, J., Thiele, I., \& Tuohy, K. (2018). Gut microbiota functions: metabolism of nutrients and other food components. In (Vol. 57, pp. 1-24): Dr. Dietrich Steinkopff Verlag GmbH and Co. KG.

Ruckert, C. (2016). Sulfate reduction in microorganisms - recent advances and biotechnological applications. Current Opinion in Microbiology, 33 , 140-146. doi:10.1016/j.mib.2016.07.007

Schuetz, R., Kuepfer, L., \& Sauer, U. (2007). Systematic evaluation of objective functions for predicting intracellular fluxes in Escherichia coli. Molecular systems biology, 3 (1), 119-119. doi:10.1038/msb4100162 
Seif, Y., Kavvas, E., Lachance, J.-C., Yurkovich, J. T., Nuccio, S.-P., Fang, X., . . . Monk, J. M. (2018). Genome-scale metabolic reconstructions of multiple Salmonella strains reveal serovar-specific metabolic traits. Nature communications, 9 (1), 1-12. doi:10.1038/s41467-018-06112-5

Simon, J., van Spanning, R. J., \& Richardson, D. J. (2008). The organisation of proton motive and nonproton motive redox loops in prokaryotic respiratory systems. Biochim Biophys Acta, 1777 (12), 1480-1490. doi:10.1016/j.bbabio.2008.09.008

Tang, K., Baskaran, V., \& Nemati, M. (2009). Bacteria of the sulphur cycle: An overview of microbiology, biokinetics and their role in petroleum and mining industries. Biochemical Engineering Journal, 44 (1), 73-94. doi:10.1016/j.bej.2008.12.011

Tang, Y., Pingitore, F., Mukhopadhyay, A., Phan, R., Hazen, T. C., \& Keasling, J. D. (2007). Pathway confirmation and flux analysis of central metabolic pathways in Desulfovibrio vulgaris Hildenborough using gas chromatography-mass spectrometry and Fourier transform-ion cyclotron resonance mass spectrometry. Journal of Bacteriology, 189 (3), 940-949. doi:10.1128/Jb.00948-06

Tatusov, R. L., Galperin, M. Y., Natale, D. A., \& Koonin, E. V. (2000). The COG database: a tool for genome-scale analysis of protein functions and evolution. Nucleic Acids Research, 28 (1), 33-36. doi:10.1093/nar/28.1.33

Thauer, R. K., Jungermann, K., \& Decker, K. (1977). Energy conservation in chemotrophic anaerobic bacteria. Bacteriological reviews, 41 (1), 100-180.

Thauer, R. K., Stackebrandt, E., \& Hamilton, A. W. (2007). Energy metabolism and phylogenetic diversity of sulphate-reducing bacteria. InSulphate-Reducing Bacteria: Environmental and Engineered Systems(pp. 1-37): Cambridge University Press.

Thiele, I., \& Palsson, B. O. (2010). A protocol for generating a high-quality genome-scale metabolic reconstruction. Nature protocols, 5 (1), 93-93. doi:10.1038/nprot.2009.203

Traore, A. S., Fardeau, M. L., Hatchikian, C. E., Legall, J., \& Belaich, J. P. (1983). Energetics of Growth of a Defined Mixed Culture of Desulfovibrio-Vulgaris and Methanosarcina-Barkeri - Interspecies Hydrogen Transfer in Batch and Continuous Cultures. Applied and Environmental Microbiology, 46 (5), 1152-1156. doi:10.1128/Aem.46.5.1152-1156.1983

Traore, A. S., Hatchikian, C. E., Belaich, J.-P., \& Gall, J. L. (1981). Microcalorimetric studies of the growth of sulfate-reducing bacteria: energetics of Desulfovibrio vulgaris growth. Journal of Bacteriology, 145 (1), 191-199.

Vita, N., Valette, O., Brasseur, G., Lignon, S., Denis, Y., Ansaldi, M., . . . Pieulle, L. (2015). The primary pathway for lactate oxidation in Desulfovibrio vulgaris. Frontiers in microbiology, 6 , 606-606. doi:10.3389/fmicb.2015.00606

Voordouw, G. (2002). Carbon monoxide cycling by Desulfovibrio vulgaris Hildenborough. Journal of Bacteriology, 184 (21), 5903-5911. doi:10.1128/jb.184.21.5903-5911.2002

Walker, C. B., He, Z., Yang, Z. K., Ringbauer, J. A., He, Q., Zhou, J., . . . Stahl, D. A. (2009). The electron transfer system of syntrophically grown Desulfovibrio vulgaris. Journal of Bacteriology, 191 (18), 5793-5801. doi:10.1128/JB.00356-09

Wang, R. (2012). Physiological Implications of Hydrogen Sulfide: A Whiff Exploration That Blossomed. Physiological Reviews, 92 (2), 791-896. doi:10.1152/physrev.00017.2011

Wood, P. M. (1978). A chemiosmotic model for sulphate respiration.FEBS letters, 95 (1), 12-18. doi:10.1016/0014-5793(78)80042-8

Wu, S., Zhu, Z., Fu, L., Niu, B., \& Li, W. (2011). WebMGA: a customizable web server for fast metagenomic sequence analysis.BMC Genomics, 12 (1), 444-444. doi:10.1186/1471-2164-12-444 
Zhou, J. Z., He, Q., Hemme, C. L., Mukhopadhyay, A., Hillesland, K., Zhou, A. F., . . . Arkin, A. P. (2011). How sulphate-reducing microorganisms cope with stress: lessons from systems biology.Nature Reviews Microbiology, 9 (6), 452-466. doi:10.1038/nrmicro2575

\section{Appendix}

\section{Abbreviations}

Ack - Acetate kinase

Acs - Acetyl-CoA synthetase

Apr - Adenosine-5'-phosphosulfate reductase

APS - Adenosine 5'-phosphosulfate

ATP - Adenosine triphosphate

ATPase - ATP synthase

DSR - Dissimilatory sulfate reduction

Dsr - Dissimilatory sulfite reductase

Ech - energy-conserving hydrogenases

FBA - Flux balance analysis

FBEB - Flavin-based electron bifurcation

$\mathrm{Fd}_{\text {red }}$ - Reduced Ferredoxin

$\mathrm{Fd}_{\mathrm{ox}}$ - Oxidized Ferredoxin

Fdh - Formate dehydrogenase

$\mathrm{Fqo}-\mathrm{F}_{420} \mathrm{H}_{2}$ : quinone oxidoreductase

GPR - gene-protein-reaction

Hase - Hydrogenase

Hdr - Heterodisulfide reductase

Hmc - High molecular weight complex

Ldh - Lactate dehydrogenase

$\mathrm{MQ}$ - Menaquinone

$\mathrm{MQH}_{2}-$ Menaquinol

$\mathrm{NAD}^{+}$- Nicotinamide adenine dinucleotide

$\mathrm{NADH}$ - Nicotinamide adenine dinucleotide, reduced form

Nfn - NADH-dependent ferredoxin:NADP reductase

Nuo - NADH: quinone oxidoreductase

Pfl - Pyruvate: formate lyase

pmf - Proton motive force

Por - Pyruvate: ferredoxin oxidoreductase 
PPi - Inorganic pyrophosphate

Pta - Phosphotransacetylase

Qrc - Quinone reductase complex

Qmo - Quinone-interacting membrane-bound oxidoreductase

Rnf - ferredoxin: NAD reductase

SLP - substrate-level phosphorylation

SRP - Sulfate-reducing prokaryotes

Tmc - Transmembrane complex

$\mathrm{TpIc}_{3}$ - Type I cytochrome $\mathrm{c}_{3}$

\section{List of Tables}

Table 1 . Sulfate-reducing prokaryotes examined in this study with sequenced genome.

\begin{tabular}{|c|c|c|c|c|c|}
\hline Organism Name & Abbreviation & NCBI assembly & Size $(\mathrm{Mb})$ & $\mathrm{GC} \%$ & Note \\
\hline Archaeoglobus fulgidus DSM 4304 & Af4304 & GCA_000008665.1 & 2.18 & 48.6 & Archaea \\
\hline Archaeoglobus fulgidus DSM 8774 & Af8774 & GCA_000734035.1 & 2.32 & 48.1 & Archaea \\
\hline Archaeoglobus profundus DSM 5631 & Apro & GCA_000025285.1 & 1.56 & 42 & Archaea \\
\hline Archaeoglobus sulfaticallidus PM70-1 & Asul & GCA_000385565.1 & 2.08 & 43.2 & Archaea \\
\hline Archaeoglobus veneficus SNP6 & Aven & GCA_000194625.1 & 1.9 & 47 & Archaea \\
\hline Desulfotomaculum ferrireducens & Dfer & GCA_002005145.1 & 3.24 & 45.4 & Gram-positive \\
\hline Desulfotomaculum nigrificans CO-1-SRB & Dnjg & GCA_000214435.1 & 2.89 & 46.6 & Gram-positive \\
\hline Desulfotomaculum reducens MI-1 & Dred & GCA_000016165.1 & 3.61 & 42.3 & Gram-positive \\
\hline Desulfotomaculum ruminis DSM 2154 & Drum & GCA_000215085.1 & 3.97 & 47.2 & Gram-positive \\
\hline Desulfovibrio africanus Walvis Bay & Dafr & GCA_000212675.2 & 4.2 & 61.4 & Gram-negative \\
\hline Desulfovibrio alaskensis G20 & Dala & GCA_000012665.1 & 3.73 & 57.8 & Gram-negative \\
\hline Desulfovibrio desulfuricans ATCC 27774 & Ddes & GCA_000022125.1 & 2.87 & 58.1 & Gram-negative \\
\hline Desulfovibrio desulfuricans ND132 & DND132 & GCA_000189295.2 & 3.86 & 65.2 & Gram-negative \\
\hline Desulfovibrio fairfieldensis CCUG 45958 & Dfai & GCA_001553605.1 & 3.7 & 60.9 & Gram-negative \\
\hline Desulfovibrio gigas ATCC 19364 & Dgig & GCA_000468495.1 & 3.8 & 63.69 & Gram-negative \\
\hline Desulfovibrio hydrothermalis AM13 & Dhyd & GCA_000331025.1 & 3.71 & 45.11 & Gram-negative \\
\hline Desulfovibrio magneticus RS-1 & Dmag & GCA_000010665.1 & 5.32 & 62.7 & Gram-negative \\
\hline Desulfovibrio piger FI11049 & Dpig & GCA_900116045.1 & 2.81 & 64.2 & Gram-negative \\
\hline Desulfovibrio salexigens DSM 2638 & & GCA_000023445.1 & 4.29 & 47.1 & Gram-negative \\
\hline Desulfovibrio sp. FW1012B & DFW & GCA_000177215.2 & 4.22 & 66.46 & Gram-negative \\
\hline Desulfovibrio vulgaris Hildenborough & $\mathrm{DvH}$ & GCA_000195755.1 & 3.77 & 63.24 & Gram-negative \\
\hline Desulfovibrio vulgaris DP4 & DvD & GCA_000015485.1 & 3.66 & 63.15 & Gram-negative \\
\hline Desulfovibrio vulgaris Miyazaki F & DvM & GCA_000021385.1 & 4.04 & 67.1 & Gram-negative \\
\hline Desulfovibrio vulgaris RCH1 & DvR & GCA_000166115.1 & 3.73 & 63.24 & Gram-negative \\
\hline
\end{tabular}

Table 2 . Average stoichiometry of cell synthesis and energy reactions for SRPs.

\begin{tabular}{lll}
\hline Electron donor & Electron acceptor & Reaction \\
\hline Lactate $^{*}$ & Sulfate & Lactate +0.47 sulfate -0.91 acetate +0.47 sulfide +0.91 carbon dioxide + Biomass \\
Lactate** $^{*}$ & Sulfate & Lactate +1.43 sulfate -1.43 sulfide +2.82 carbon dioxide + Biomass
\end{tabular}




\begin{tabular}{lll}
\hline Electron donor & Electron acceptor & Reaction \\
\hline $\mathrm{H}_{2}$ & Sulfate & $\mathrm{H}_{2}+0.25$ sulfate +0.033 Acetate +0.031 carbon dioxide -0.25 sulfide + Biomass \\
\hline
\end{tabular}

*For Desulfovibrio and Desulfotomaculum exceptD.magneticus and D. gigas.

**For Archaeoglobus .

Table 3 . Redox potentials of the electron donors or acceptors important in SRPs, adapted from (Lundblad \& Macdonald, 2018; R. K. Thauer et al., 1977)

\begin{tabular}{ll}
\hline Redox couple & $\mathrm{E}^{\circ}(\mathrm{mV})$ \\
\hline $\mathrm{SO}_{4}{ }^{2-} / \mathrm{HSO}_{3}{ }^{-}$ & -516 \\
$\mathrm{APS} / \mathrm{AMP}^{-}+\mathrm{HSO}_{3}{ }^{-}$ & -60 \\
$\mathrm{HSO}_{3}{ }^{-} / \mathrm{HS}^{-}$ & -116 \\
Pyruvate$/$ lactate $^{-}$ & -190 \\
${\text { Acetyl-CoA }+\mathrm{CO}_{2} / \text { pyruvate }}^{-}$ & -498 \\
$\mathrm{H}^{+} / \mathrm{H}_{2}$ & -414 \\
$\mathrm{CO}_{2} /$ formate & -270 to -300$)^{*}$ \\
$\mathrm{MQ}^{*} \mathrm{MQH}_{2}$ & -432 \\
$\mathrm{TpIc}_{3}$ ox/red & -74 \\
$\mathrm{Fd}_{\text {red }} / \mathrm{Fd}_{\text {ox }}$ & -205 to -290 \\
$\mathrm{NAD}^{+} / \mathrm{NADH}$ & -398 \\
$\mathrm{NADP}^{+} / \mathrm{NADPH}$ & -320 \\
\hline
\end{tabular}

*Calculated for a $\mathrm{H}_{2}$ partial pressure of $1 \mathrm{~Pa}$ and $10 \mathrm{~Pa}$, respectively

\section{Hosted file}

Figures_v3.docx available at https://authorea.com/users/354593/articles/478109-comparativemetabolic-modeling-of-multiple-sulfate-reducing-prokaryotes-reveals-versatile-energyconservation-mechanisms 


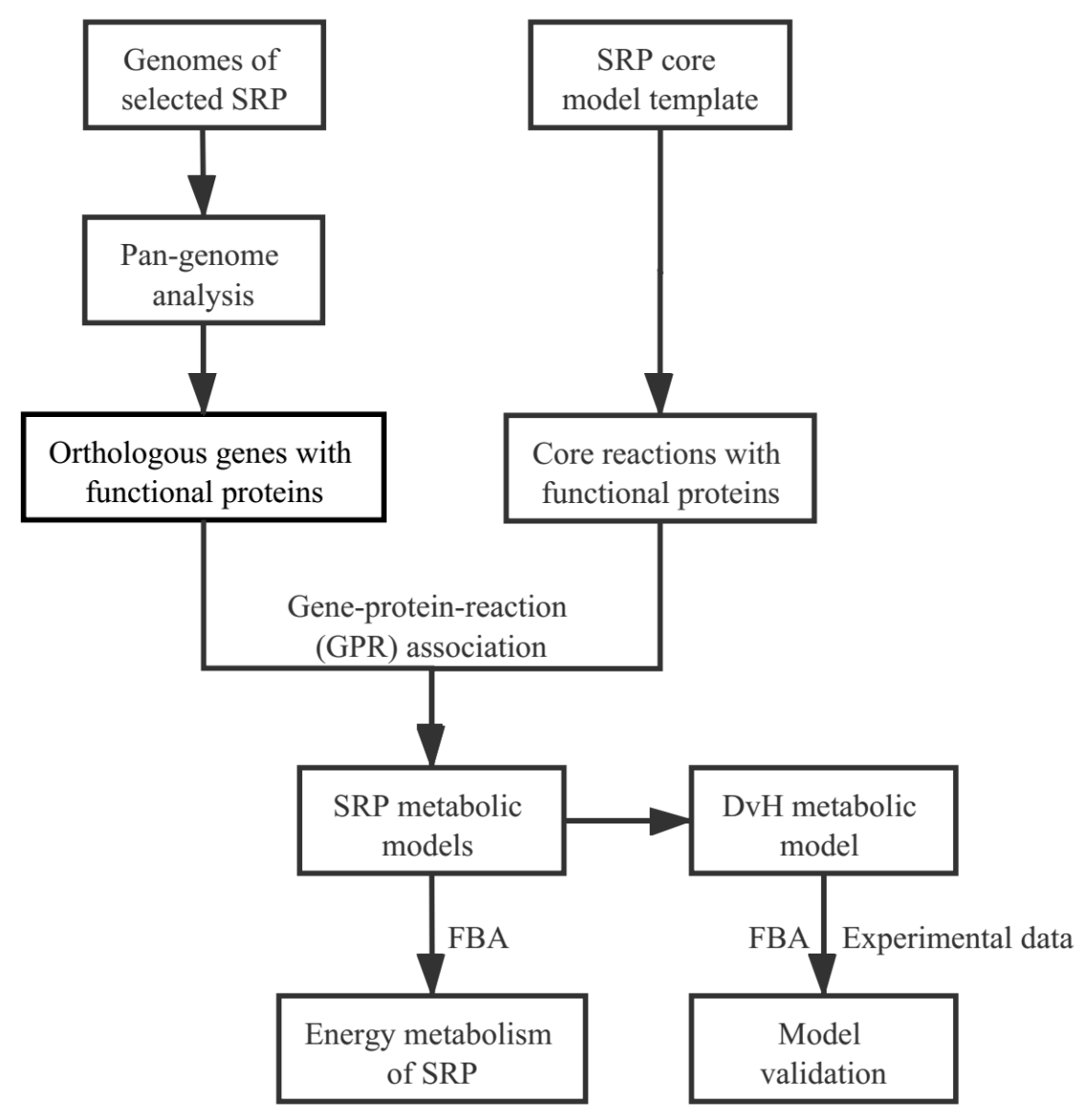



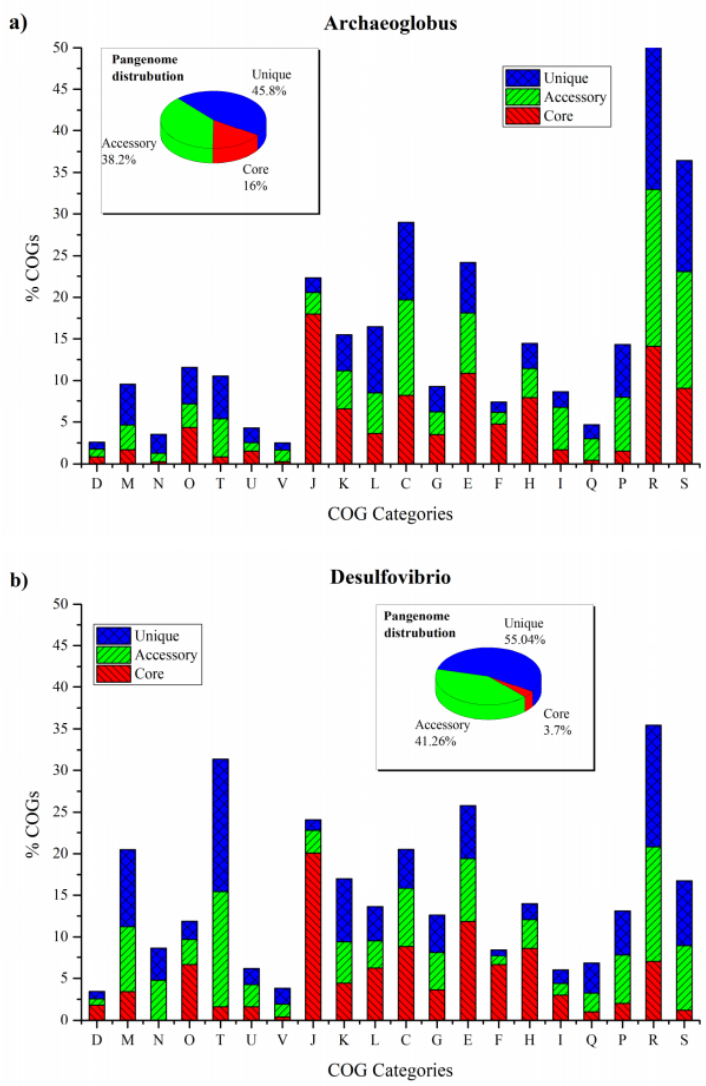


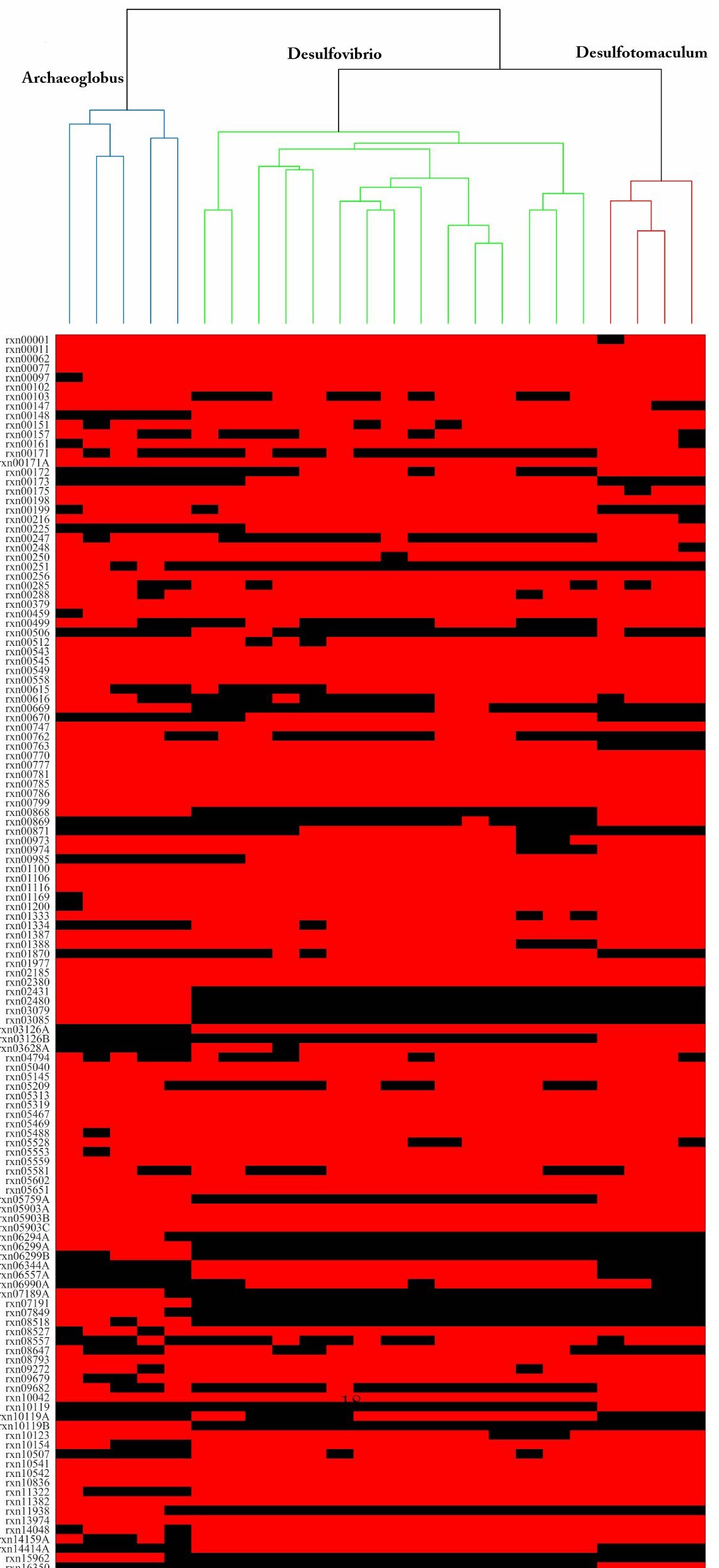



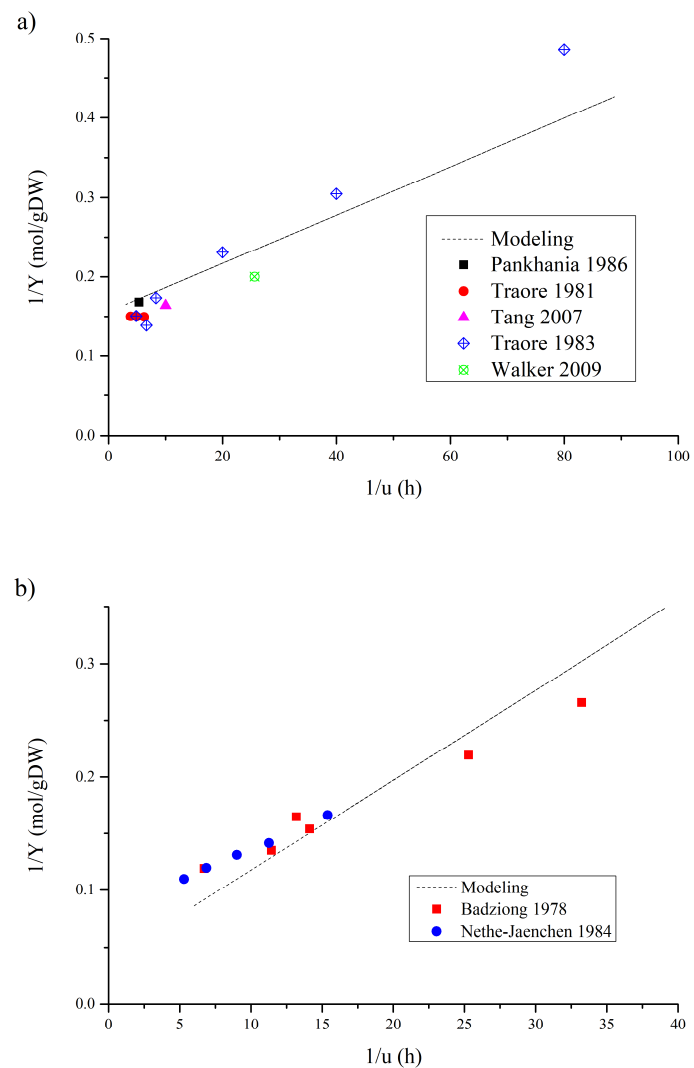


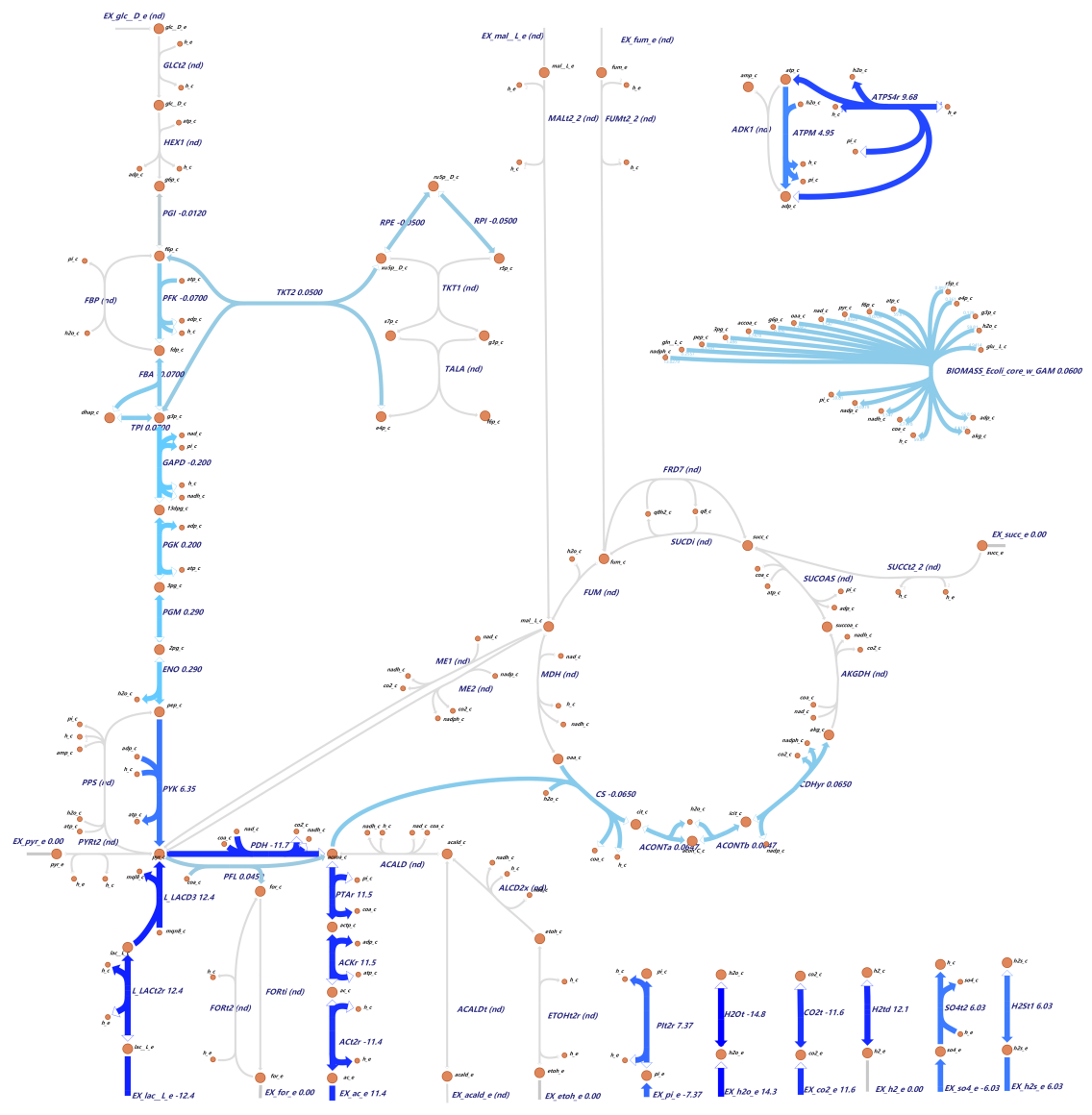




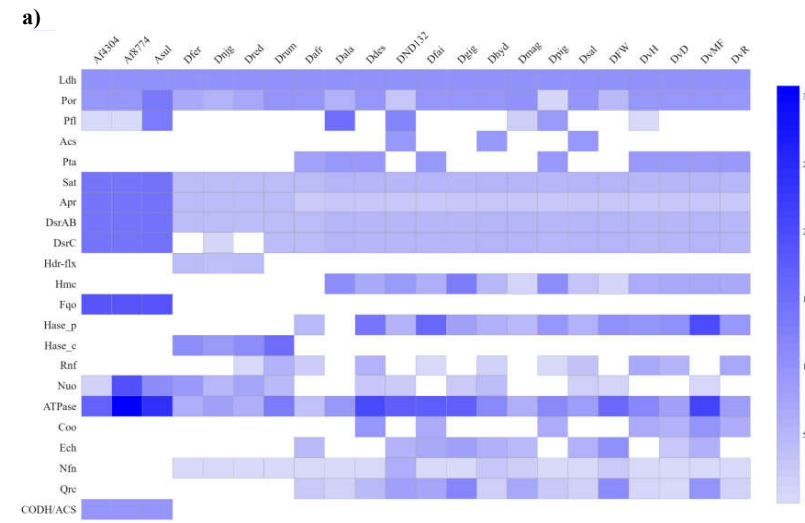

b)

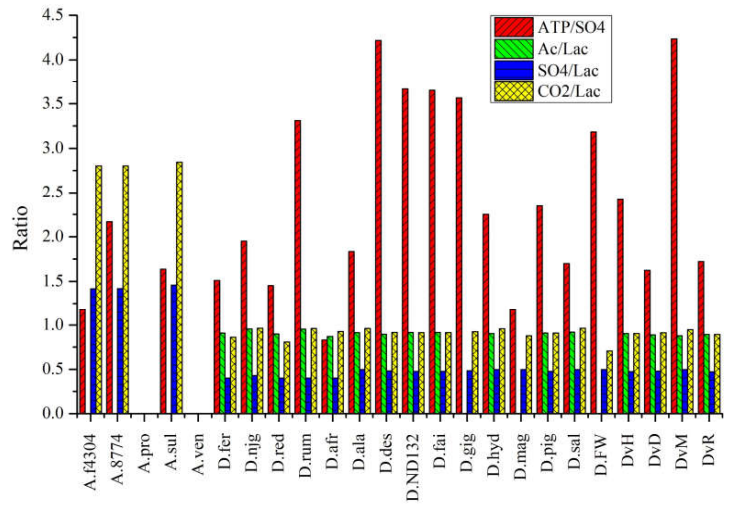




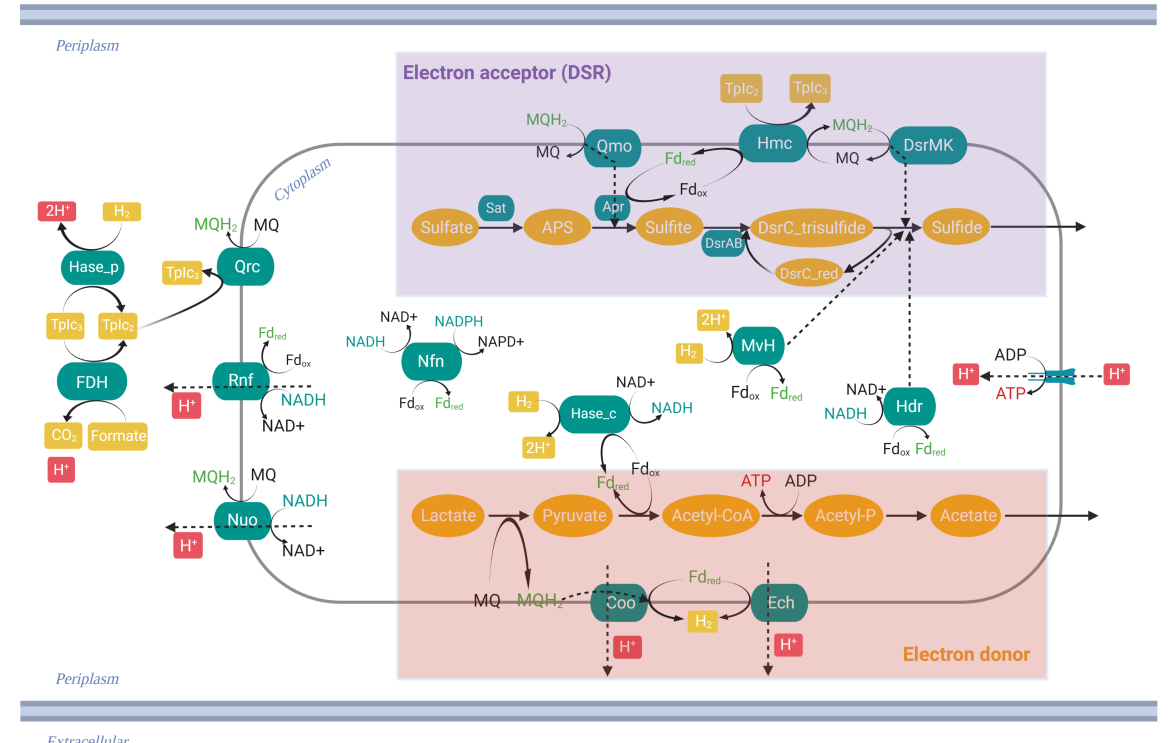




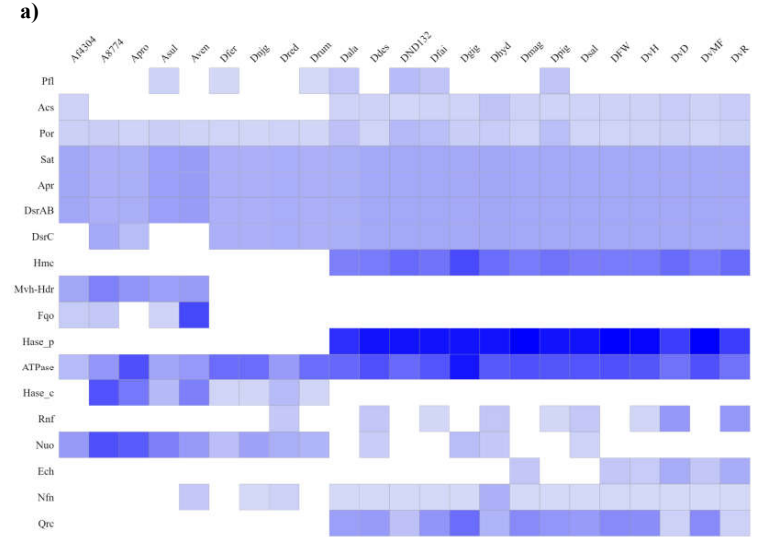

b)

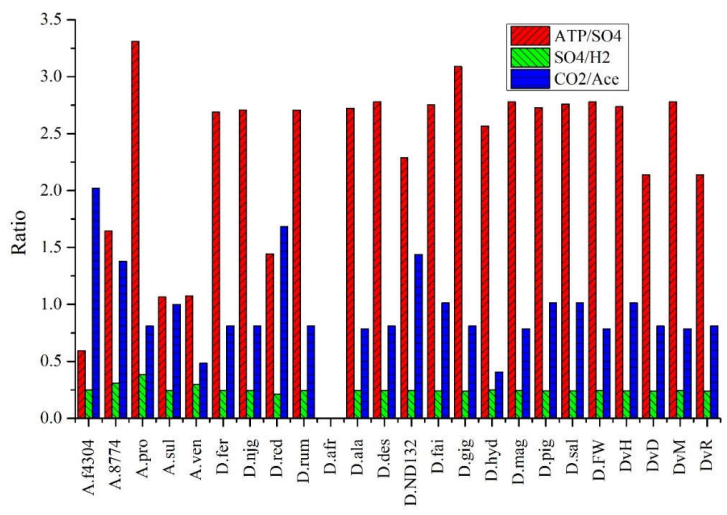




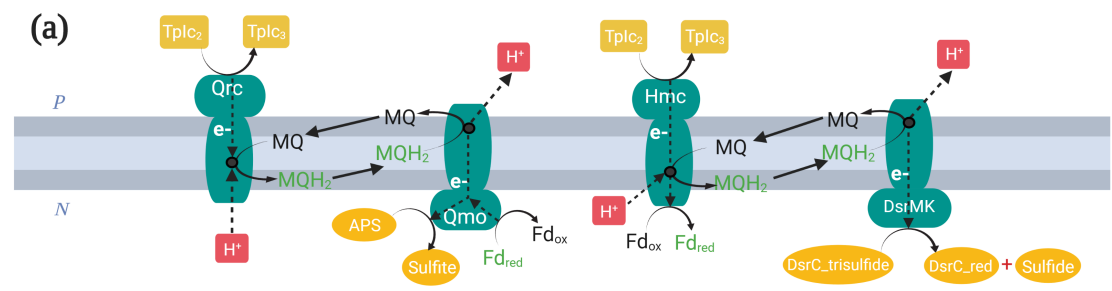

(b)

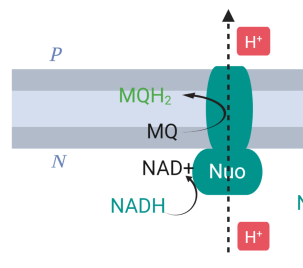

(d)

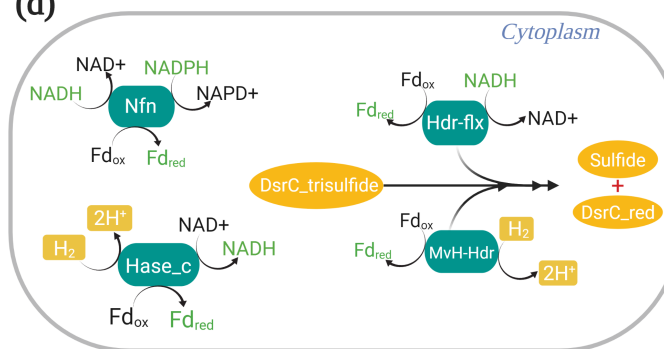

(c)

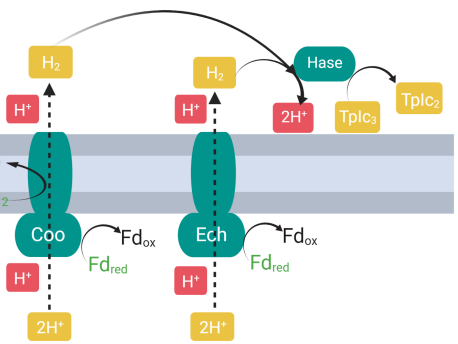

